\title{
A ICTIOFAUNA DA BACIA DO RIO SARAPUÍ, SP, BRASIL: ESTRUTURA DAS ASSEMBLEIAS E A INFLUÊNCIA DE DIFERENTES VARIÁVEIS AMBIENTAIS
}

\author{
NASCIMENTO, M. B. ${ }^{1} \&$ SMITH, W. S. ${ }^{1},{ }^{2 *}$ \\ 1. Laboratório de Ecologia Estrutural e Funcional - Universidade Paulista, campus Sorocaba/SP \\ 2. Programa de Pós Graduação em Ciências da Engenharia Ambiental, Centro de Recursos Hídricos \\ e Ecologia Aplicada, USP, São Carlos, SP, Brasil \\ *Corresponding author: welber_smith@uol.com.br
}

\begin{abstract}
Nascimento, M. B. \& Smith, W. S., (2016). A ictiofauna da bacia do rio Sarapuí, SP, Brasil: estrutura das assembleias e a influência de diferentes variáveis ambientais. Braz. J. Aquat. Sci. Technol. 20(1). elSSN 1983-9057. DOI: 10.14210/bjast.v20n1. This manuscript aimed to determine the ichthyofauna composition from Sarapuí River Basin, verifying the influence of environmental characteristics related to seasonality and margin condition, thus, fish were collected in four sampling sites in the main river, two in the Camapuã stream and 2 in the Macacos stream, in addition to previous work. Twenty-seven species, distributed in thirteen families and four orders was identified. Characiformes was the most representative order, outstanding the Characidae family and Astyanax altiparanae specie. The sampling sites Sa01 and $\mathrm{Sa} 03$ was the most representative in species number, site $\mathrm{Sa} 04$ was the most representative in abundant species, while the diversity was bigger in Sa03. Considering all the work already done was verified the occurrence of 61 species belonging to 19 families and 6 orders, and the orders Characiformes and Siluriformes was the most representative. This study improves information about the fish assemblages of Sarapuí River Basin and allow to conclude that the seasonality influence fish assemblage in the main river and in the tributaries, and that abundance is an important descriptor associated with richness, and the size of the watercourse represented in this study by the width influence the abundances of species. Furthermore, the variables that express the conditions the margin has low influence on the community descriptors used.
\end{abstract}

Key words: Community, Species diversity, Anthropogenic impacts, Sazonality, Inventory.

\section{INTRODUÇÃO}

As bacias hidrográficas apresentam inúmeros ecossistemas aquáticos que fornecem serviços ambientais tais como a água e a biodiversidade. Os rios desempenham um papel fundamental nos ecossistemas, fornecendo uma gama de funções, tais como abrigo e fonte de alimento para uma série de espécies biológicas, ajudam na regulação de inundações e servem como refúgio ecológico (Hoelzl, 2007). Além disso, possuem alta diversidade de fauna e flora aquática, possibilitando a heterogeneidade da ictiofauna dulcícola (Buckup \& Menezes 2007).

Os usos e ocupação da bacia hidrográfica e da sua respectiva malha hídrica, inserem modificações acentuadas no sistema e nas comunidades biológicas. Segundo Walsh et al. (2005) os rios que são severamente afetados, devido à influência antrópica sofrem da síndrome do rio urbano. Uma forma possível para superar a síndrome do rio urbano é gerenciar a saúde do rio de forma eficaz através da compreensão das interações de fatores antrópicos e naturais, particularmente por meio de um programa de monitoramento de custo-benefício bem planejado. Distúrbios antrópicos incluem canalização, barragens, remoção da vegetação ripária, lançamento de esgoto e a presença de espécies invasoras (Santos et al., 2011).

Os rios se formam e se transformam influenciados pelo ciclo hidrológico envolvendo a atmosfera e o solo (Oleriano \& Dias, 2007). Correspondem ao sistema de chuvas e vazantes geradoras de futuras modificações naturais ao longo de seu curso, onde em áreas planas o curso do rio desvia dos obstáculos enquanto em áreas com declive tenta erodir e transpor os obstáculos, assim também destaca por profundidade, largura e extensão, onde correntes volumosas de água se deslocam na superfície terrestre, sempre das áreas mais elevadas para as menos elevadas, unindo as águas dos afluentes e subafluentes, podendo assim desembocar em outro rio (Barrella et al., 2000).

Os ecossistemas lênticos correspondem aos lagos, lagoas, represas e açudes e em particular as lagoas marginais (Smith, 2003). O rio Sarapuí apresenta inúmeras áreas com lagoas marginais. Estas são formadas principalmente no período chuvoso quando o rio invade áreas mais baixas, transformando em viveiros naturais de peixes, pois são áreas importantes de alimentação e abrigo, neste caso através das macrófitas aquáticas (Smith \& Barrela, 2000). Apesar disso, algumas lagoas marginais são perenes e somente permanecem no período de cheia, dependendo do grau de conexão com o rio. 
Importante também são as matas ciliares para a manutenção do regime hídrico, fornecimento de recursos para a ictiofauna e manutenção da estabilidade dos ambientes, e quando são suprimidas põem em risco os habitats (Ferreira \& Casatti, 2006). Cabe salientar que os galhos, troncos e folhas depositadas no leito do rio proveniente da mata ciliar contribuem para o abrigo e alimentação de diferentes espécies de peixes (Barrella et al., 2000).

Pelo fato do rio ser um sistema aberto, de fluxo contínuo da nascente à foz cujas características vão se alterando ao longo do seu curso, as atividades antrópicas representam sérias ameaças à diversidade de peixes (Uieda \& Castro, 2002; Dala-Corte et al., 2009). Esta degradação ambiental justifica a necessidade de estudos que avaliem tais impactos e proponham medidas de proteção e conservação (Hoffman et al., 2005, Marinho et al., 2006). As principais ameaças à ictiofauna estão relacionadas com a poluição, desmatamento, barragens, pesca predatória e introdução de espécies exóticas (Agostinho et al., 2005).

No Brasil, já foram catalogadas 2.587 espécies (Buckup \& Menezes, 2007) e segundo Langeani et al. (2007) o Alto Paraná abriga 310 espécies de peixes, distribuᄀídas em 11 ordens e 38 famílias. Para o Estado de São Paulo, Castro e Menezes (1998) citam a ocorrência de 166 espécies. Porém, em artigo mais recente (Oyakawa \& Menezes, 2011) destaca-se que para o estado de São Paulo existem cerca de 391 espécies, o que corresponde a aproximadamente $15 \%$ do total estimado para todo o território brasileiro. Deste total, 260 espécies ocorrem no Alto Paraná, 97 no Ribeira de Iguape, 71 no Paraíba do Sul e 57 na Bacia Litorânea, sendo que algumas espécies podem ocorrer em duas ou mais bacias. As espécies estão distribuídas em 10 ordens e 39 famílias, verificando-se que Characidae (com 83) e Loricariidae (com 81) são as mais numerosas.

Com relação à bacia do rio Sorocaba, onde está inserido o rio Sarapuí e seus afluentes, já foram registradas até o presente momento 71 espécies, as quais se encontram distribuídas em 22 famílias e 7 ordens, destacando a ordem Characiformes (Smith et al., 2007). A fim de ampliar o conhecimento da ictiofauna, o presente trabalho teve como objetivo caracterizar a comunidade de peixes da bacia hidrográfica do Rio Sarapuí, verificando a influência de características ambientais relacionadas a sazonalidade e condição das margens. De forma a complementar as informações decorrentes da pesquisa, também foi realizada uma compilação dos resultados obtidos em estudos anteriores realizados na bacia do rio Sarapuí.

\section{MATERIAL E MÉTODOS}

O rio Sarapuí, conhecido como Sarapus e de origem indígena "çarapó-y", que significa rio dos sarapós - espécie de peixe escorregadio nasce na cidade de Piedade e segundo Smith \& Petrere (2000), corta as cidades de Pilar do Sul, Salto de Pirapora, Araçoiaba da Serra, Alambari, Capela do Alto, Itapetininga, Tatuí e Iperó (Figura 1) desaguando na margem esquerda do rio Sorocaba. A bacia é caracterizada por Cerrado e Mata Atlântica, com clima tropical (IBGE, 2009) e pertence à bacia hidrográfica do rio Sorocaba, Unidade Hidrográfica do Estado de São Paulo (URGHI 10).

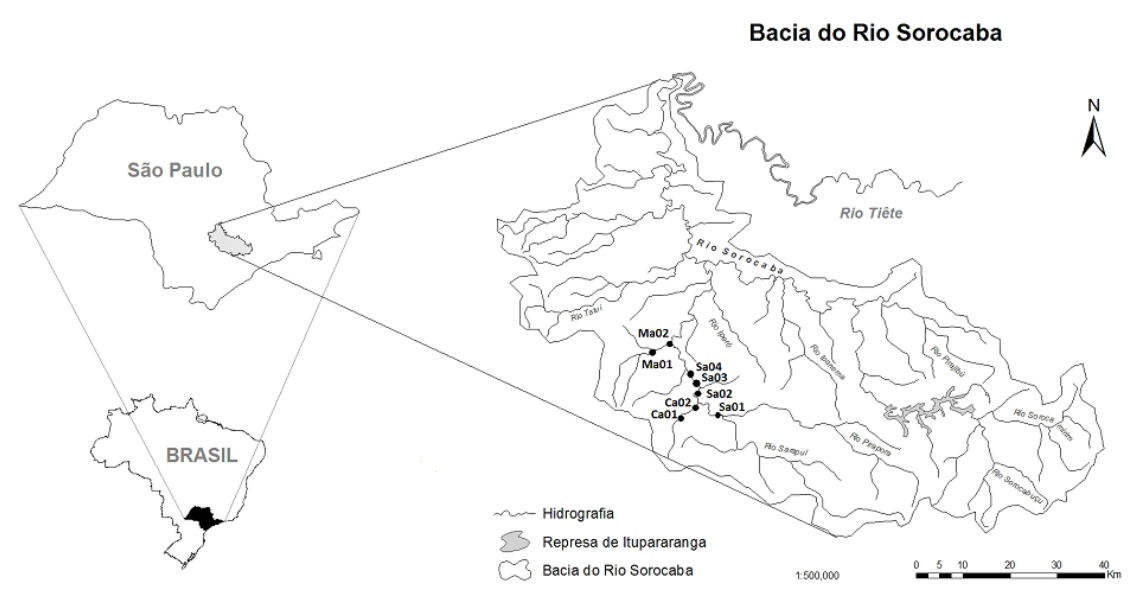

Figura 1 - Localização do rio Sarapuí, afluentes e pontos de coleta utilizados pelo presente estudo (organizado por Bruno Mello). 
As coletas foram realizadas nas épocas chuvosa e seca, período correspondente a janeiro e julho do ano de 2010, em 8 pontos diferentes, sendo 4 no rio Sarapuí, 2 no Ribeirão do Camapuã e 2 no Ribeirão dos Macacos (Tabela 1). Para caracterizar os pontos de coleta foram obtidos dados sobre a temperatura da água, a largura, a profundidade e os possíveis impactos ambientais presentes em cada ponto de coleta. A temperatura da água foi medida com termômetro digital a fim de caracterizar o regime climático, enquanto que a largura e a profundidade foram medidas com trena. Além disso, foram obtidos os dados de índice pluviométrico através do banco de dados meteorológicos do Instituto Nacional de Meteorologia (INMET).

Tabela 1 - Pontos de coleta no rio Sarapuí e afluentes e suas respectivas coordenadas geográficas.

\begin{tabular}{|c|c|c|c|c|c|}
\hline Pontos & Rio / Afluentes & Município & Coordenadas & & Impactos \\
\hline $\mathrm{Sa} 01$ & Sarapuí & Araçoiaba da Serra & $23^{\circ} 35^{\prime} 21^{\prime \prime S}$ & $47^{\circ} 45^{\prime} 77^{\prime \prime} \mathrm{W}$ & Pastagem \\
\hline $\mathrm{Sa} 02$ & Sarapuí & Araçoiaba da Serra & $23^{\circ} 35^{\prime} 41^{\prime \prime S}$ & $47^{\circ} 35^{\prime} 98^{\prime \prime} \mathrm{W}$ & Pastagem \\
\hline $\mathrm{Sa} 03$ & Sarapuí & Araçoiaba da Serra & $23^{\circ} 36^{\prime} 58^{\prime \prime} \mathrm{S}$ & $47^{\circ} 44^{\prime} 65^{\prime \prime} \mathrm{W}$ & Extração de areia \\
\hline $\mathrm{Sa} 04$ & Sarapuí & Araçoiaba da Serra & $23^{\circ} 34^{\prime} 99^{\prime \prime S}$ & $47^{\circ} 45^{\prime} 60^{\prime \prime} \mathrm{W}$ & Extração de areia \\
\hline $\mathrm{Ca} 01$ & Ribeirão do Camapuã & Sarapuí & $23^{\circ} 36^{\prime} 65^{\prime \prime} \mathrm{S}$ & $47^{\circ} 45^{\prime} 79^{\prime \prime} \mathrm{W}$ & Pastagem - Agricultura \\
\hline $\mathrm{Ca} 02$ & Ribeirão do Camapuã & Sarapuí & $23^{\circ} 36^{\prime} 73^{\prime \prime S}$ & $47^{\circ} 45^{\prime} 71^{\prime \prime} \mathrm{W}$ & Pastagem - Agricultura \\
\hline Ma01 & Ribeirão dos macacos & Sarapuí & $23^{\circ} 36^{\prime} 14^{\prime \prime S}$ & $47^{\circ} 47^{\prime} 98^{\prime \prime} \mathrm{W}$ & Pastagem - Agricultura \\
\hline $\mathrm{Ma} 02$ & Ribeirão dos macacos & Sarapuí & $23^{\circ} 33^{\prime} 22^{\prime \prime S}$ & $47^{\circ} 47^{\prime} 12^{\prime \prime} \mathrm{W}$ & Pastagem - Agricultura \\
\hline
\end{tabular}

Para a captura de peixes foram utilizadas duas baterias de redes de espera com 08 dimensões diferentes de malhas (03 a $12 \mathrm{~cm}$ ), entre nós opostos, de 10 metros de comprimento e $1,5 \mathrm{~m}$ de largura, instaladas no final da tarde e retiradas na manhã seguinte de forma a permanecerem por 12 horas. Além das redes de espera, foi utilizada peneira de malha de 2 $\mathrm{mm}$ nas bordas dos respectivos pontos, próximo a margem, para melhor amostrar as espécies de menor porte, aumentando, desta forma, as possibilidades de captura de diferentes espécies e diminuindo a seletividade das mesmas (Barrela, 1989).

Os exemplares capturados foram fixados em formalina $10 \%$, transferidos ao laboratório da Universidade Paulista (UNIP), campus de Sorocaba, onde foram pesados em balança eletrônica e medidos com paquímetro, identificados utilizando Smith et al. (2003) e Smith (2003), separados em vidros rotulados com data e local da coleta e conservados em álcool $70 \%$, permanecendo na coleção do museu de zoologia da UNIP. As coletas foram autorizadas pelo ICMBio através da licença 24151-1 emitida em 21/06/2010.

Para cada ponto de coleta foi calculada a diversidade de espécies, utilizado o índice de Shannon-Wiener, com o auxílio do software PAST (Palaentological Statistics). Foi realizada uma Análise de Componentes Principais (Manly, 1986; Digby \& Kempton, 1987) utilizando o programa Past (Hammer et al., 2001), para verificar a relação entre as variáveis ambientais e biológicas avaliadas.

\section{RESULTADOS}

A caracterização ambiental dos pontos de coletas mostra nítida diferença na temperatura entre as épocas chuvosa (verão) e seca (inverno) com temperaturas variando entre $23^{\circ} \mathrm{C} \mathrm{e} 30^{\circ} \mathrm{C}$ no período chuvoso e entre $13^{\circ} \mathrm{C} \mathrm{e} 25^{\circ} \mathrm{C}$ no período seco (Figura 2). A largura nos locais amostrados variou entre 1,0 e 7,0 metros e a profundidade entre 0,5 e 3,0 metros, sendo os maiores valores registrados na época chuvosa. Deve ser ressaltando que, janeiro de 2010 foi o mês mais chuvoso em mais de 10 anos (INMET) e com isso, o nível do rio permaneceu alto, com a média de $2 \mathrm{~m}$ de profundidade e $5 \mathrm{~m}$ de largura, conforme constatado no decorrer das coletas. Nos pontos amostrados, atividades agrícolas, extração de areia e pastagem dominam a paisagem (Tabela 1).
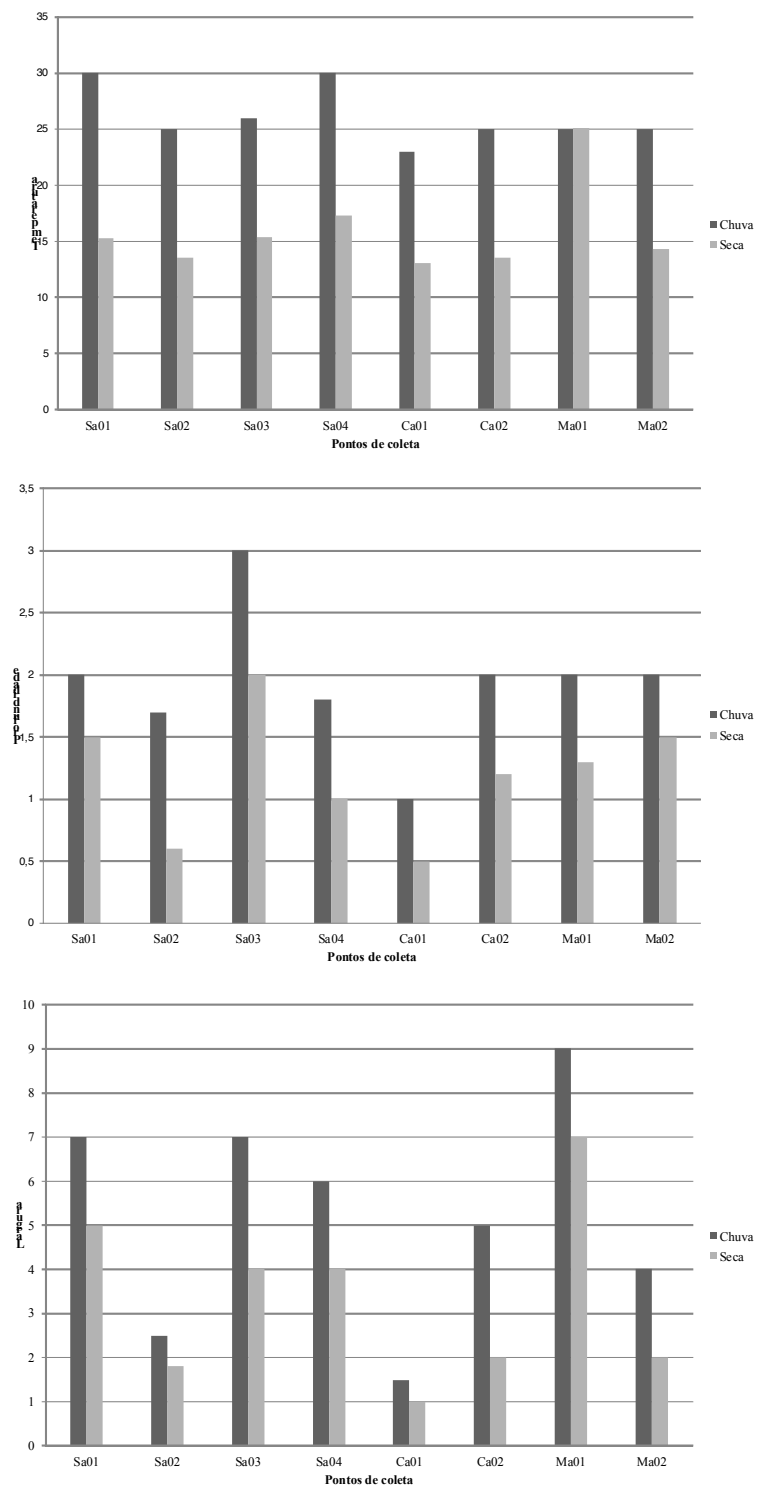

Figura 2 - Comparação dos valores de temperatura $\left({ }^{\circ} \mathrm{C}\right)$, largura e profundidade (metros) nos pontos do rio Sarapuí e afluentes estudados nas épocas chuvosa e seca. 
Ao todo foram identificadas 27 espécies de peixes pertencentes a 13 famílias e 04 ordens, sendo 22 espécies no rio Sarapuí, 9 espécies no ribeirão do Camapuã e 8 espécies no ribeirão dos Macacos
(Tabela 2). A ordem predominante foi Characiformes com 18 espécies (67\%) e Siluriformes com 8 espécies $(29,6 \%)$.

Tabela 2 - Espécies de peixes ocorrentes na Bacia do rio Sarapuí e afluentes, indicando os locais de ocorrência considerando os trabalhos publicados até o presente momento.

\begin{tabular}{|c|c|c|c|c|c|}
\hline Taxon & $\begin{array}{c}\text { Alto } \\
\text { Sarapuí }\end{array}$ & $\begin{array}{c}\text { Médio } \\
\text { Sarapuí }{ }^{1,4}\end{array}$ & $\begin{array}{c}\text { Baixo } \\
\text { Srapuí }{ }^{1}\end{array}$ & $\begin{array}{l}\text { Ribeirão dos } \\
\text { Macacos }^{1,4,5}\end{array}$ & $\begin{array}{c}\text { Ribeira } \\
\text { Camapuã }\end{array}$ \\
\hline \multicolumn{6}{|l|}{$\begin{array}{l}\text { CHARACIFORMES } \\
\text { Characidae }\end{array}$} \\
\hline Astyanax paranae & $\bullet$ & & & & \\
\hline Astyanax altiparanae & $\bullet$ & $\bullet$ & $\bullet$ & $\bullet$ & $\bullet$ \\
\hline Astyanax eignmanniorum & $\bullet$ & $\bullet$ & $\bullet$ & & \\
\hline Astyanax fasciatus & $\bullet$ & $\bullet$ & $\bullet$ & $\bullet$ & \\
\hline Astyanax scabripinnis & & & & & $\bullet$ \\
\hline Astyanax schubarti & & $\bullet$ & $\bullet$ & & \\
\hline Astyanax sp. & & & & & $\bullet$ \\
\hline Moenkhausia intermedia & & $\bullet$ & $\bullet$ & & \\
\hline Bryconamericus iheringii & $\bullet$ & & & & \\
\hline Bryconamericus stramineus & & $\bullet$ & $\bullet$ & & $\bullet$ \\
\hline Bryconamericus microcephalus & $\bullet$ & & & & \\
\hline Hyphessobrycon bifasciatus & $\bullet$ & & & & \\
\hline Hyphenobrycon eques & & $\bullet$ & $\bullet$ & & \\
\hline Hyphessobrycon anisitsi & $\bullet$ & & & & \\
\hline Piabina argentea & $\bullet$ & $\bullet$ & $\bullet$ & & \\
\hline Galeocharax krerii & & & & & $\bullet$ \\
\hline \multicolumn{6}{|l|}{$\begin{array}{l}\text { Crenuchidae } \\
\text { Characidium zebra }\end{array}$} \\
\hline Characidium gomesi & $\bullet$ & & & & \\
\hline Characidium oiticicai & $\bullet$ & & & & \\
\hline \multicolumn{6}{|l|}{ Acestrorhynchidae } \\
\hline Acestrorhynchus lacustris & & $\bullet$ & $\bullet$ & $\bullet$ & \\
\hline \multicolumn{6}{|l|}{ Curimatidae } \\
\hline Steindachnerina insculpta & & $\bullet$ & $\bullet$ & $\bullet$ & $\bullet$ \\
\hline $\begin{array}{l}\text { Cyphocharax modestus } \\
\text { Erythrinidae }\end{array}$ & & $\bullet$ & $\bullet$ & & \\
\hline $\begin{array}{l}\text { Hoplias malabaricus } \\
\text { Parodontidae }\end{array}$ & $\bullet$ & $\bullet$ & $\bullet$ & $\bullet$ & $\bullet$ \\
\hline Apareiodon piracicabae & & $\bullet$ & $\bullet$ & • & \\
\hline Apareiodon ibitiensis & $\bullet$ & & & & \\
\hline Parodon nasus & $\bullet$ & & & & \\
\hline \multicolumn{6}{|l|}{ Prochilodontidae } \\
\hline $\begin{array}{l}\text { Prochilodus lineatus } \\
\text { Anostomidae }\end{array}$ & & $\bullet$ & $\bullet$ & $\bullet$ & \\
\hline Schizodon nasutus & & - & - & & \\
\hline
\end{tabular}


Leporinus obtusidens

Salminidae

Salminus hilarii

Serrasalmidae

Serrasalmus spilopleura

GYMNOTIFORMES

Gymnotidae

Gymnotus carapo

Gymnotus sylvius

Eigenmannia virescens

\section{SILURIFORMES}

\section{Callichthyidae}

Callichthys callichthys

Hoplostenum littorale

Corydoras aeneus

\section{Loricariidae}

Hypostomus ancistroides Hypostomus margaritiffer Hypostomus variipictus

Hypostomus sp.

Neoplecostomus sp.

Rineloricaria latirostris

Rineloricaria sp. 1

Rineloricaria sp. 2

Heptapteridae Iheringichthys labrosus

Pimelodus maculatus

Rhamdia quelen

Pimelodella sp.

Pimelodella avanhandavae

Imparfinis schubarti

Imparfinis mirini

Cetopsorhamdia iheringi

Doradidae

Rhinodoras dorbignyi

Trichomycteridae

Trichomycterus iheringi

PERCIFORMES

Cichlidae

Geophagus brasiliensis

Australoheros facetus

\section{CYPRINODONTIFORMES}

\section{Poecilidae}

Phalloceros cf. harpagos

Phalloceros reisi

Phalloceros caudimaculatus

\section{SYNBRANCHIFORMES}

Synbranchidae

Synbranchus marmoratus

$$
\text { TOTAL }
$$

$\bullet$
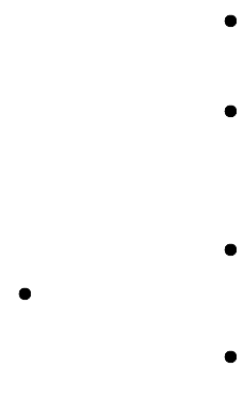

$\bullet$

$\bullet$

$\bullet$
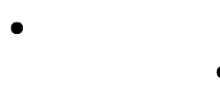

-

$\bullet$

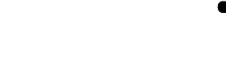

$\bullet$

$\bullet$

$\bullet$

$\bullet$

-

$\bullet$

-

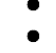

$\bullet$
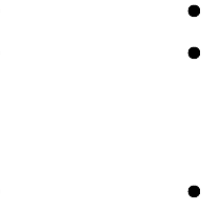

-

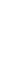

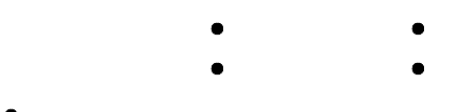

$\bullet$

$\bullet$

•
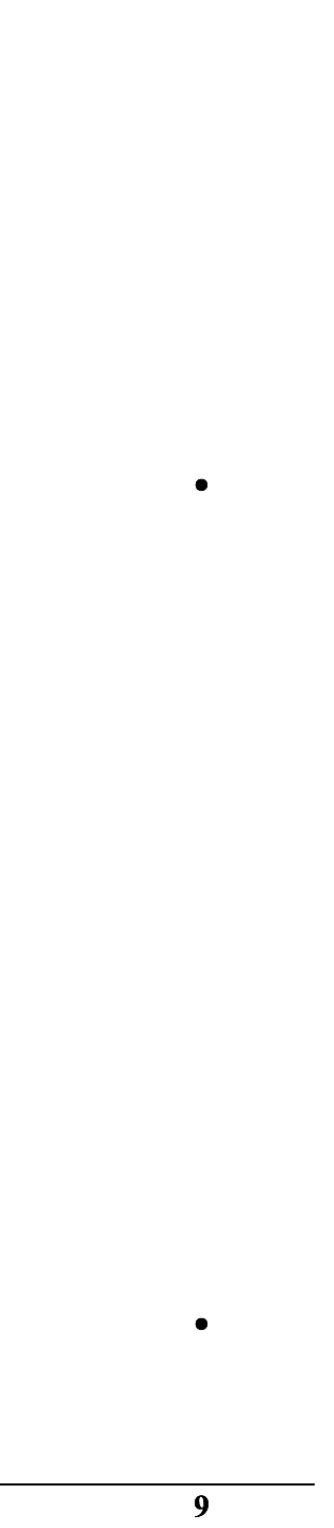

Segundo: ${ }^{1}$ Smith (1999), Smith et al. (2007) e Smith et al. (2009); ${ }^{2}$ Teshima (2010); ${ }^{3}$ Cetra et al. (2012);

${ }^{4}$ Biagioni \& Smith (2012) e ${ }^{5}$ coletas do presente trabalho. 
A Tabela 2 apresenta um inventário considerando todos os trabalhos realizados até o momento na bacia do rio Sarapuí, incluindo os dados do presente estudo. Pode-se constatar a ocorrência de 61 espécies distribuídas em 6 ordens e 19 famílias, sendo as ordens Characiformes e Siluriformes e as famílias
Characidae e Heptapteridae as mais representativas. A Figura 3 mostra os pontos inventariados até o presente momento, considerando todos os trabalhos realizados e publicados, totalizando 11 localidades da bacia do rio Sarapuí.

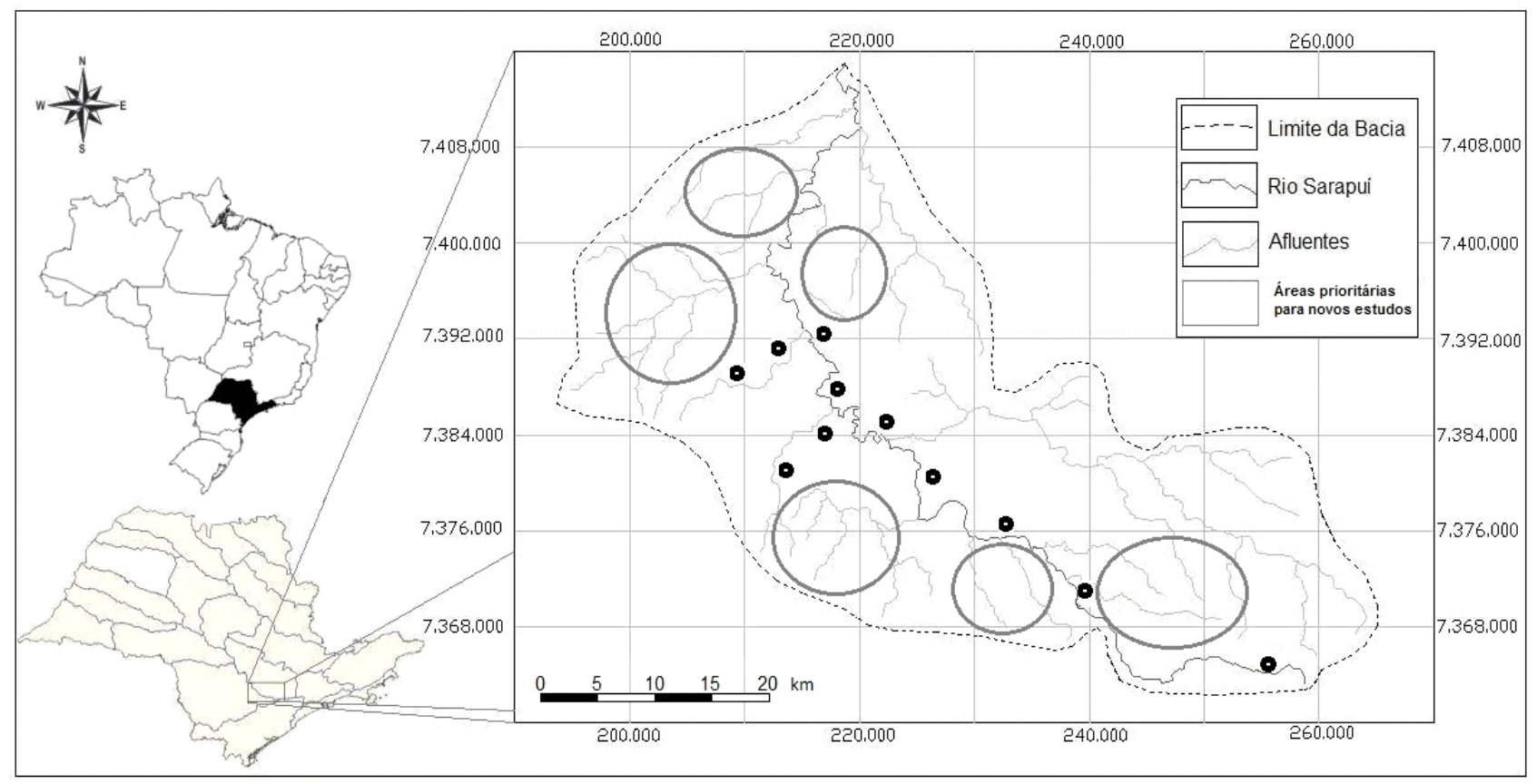

Figura 3 - Bacia do rio Sarapuí com a localização dos pontos amostrados pelos trabalhos realizados até o presente momento e as áreas prioritárias para novos estudos. Mapa modificado de Teshima (2010).

As espécies mais abundantes foram: Astyanax altiparanae e Steindachnerina inscuplta. Além disso, pode ser verificado que as espécies Astyanax altiparanae, Hoplias malabaricus e Steindachnerina inscuplta foram aquelas com distribuição mais ampla.

A riqueza variou de 3 a 12 espécies entre as estações amostradas, enquanto que a abundância variou entre 12 e 74 indivíduos (Tabela 3). Os pontos SA01, SA02 e SA03 apresentaram os maiores valores de riqueza enquanto que SA01, SA03, SA04 e MA01 apresentaram as maiores abundâncias (Figura 4). Considerando a sazonalidade na maioria dos pontos foi na época seca as maiores riquezas. Os pontos SA01 e SA02 apresentaram no período chuvoso a maior riqueza de espécies e o ponto SA03, no período seco (Figura 4). Em relação ao índice de diversidade de Shannon-Wiener ( $H^{\prime}$ ') os pontos SA01, SA02, SA03, SA04 e CA02 foram os que apresentaram os maiores valores (Figura 4).

Tabela 3 - Espécies de peixes coletadas no rio Sarapuí e afluentes na estação chuvosa e seca.

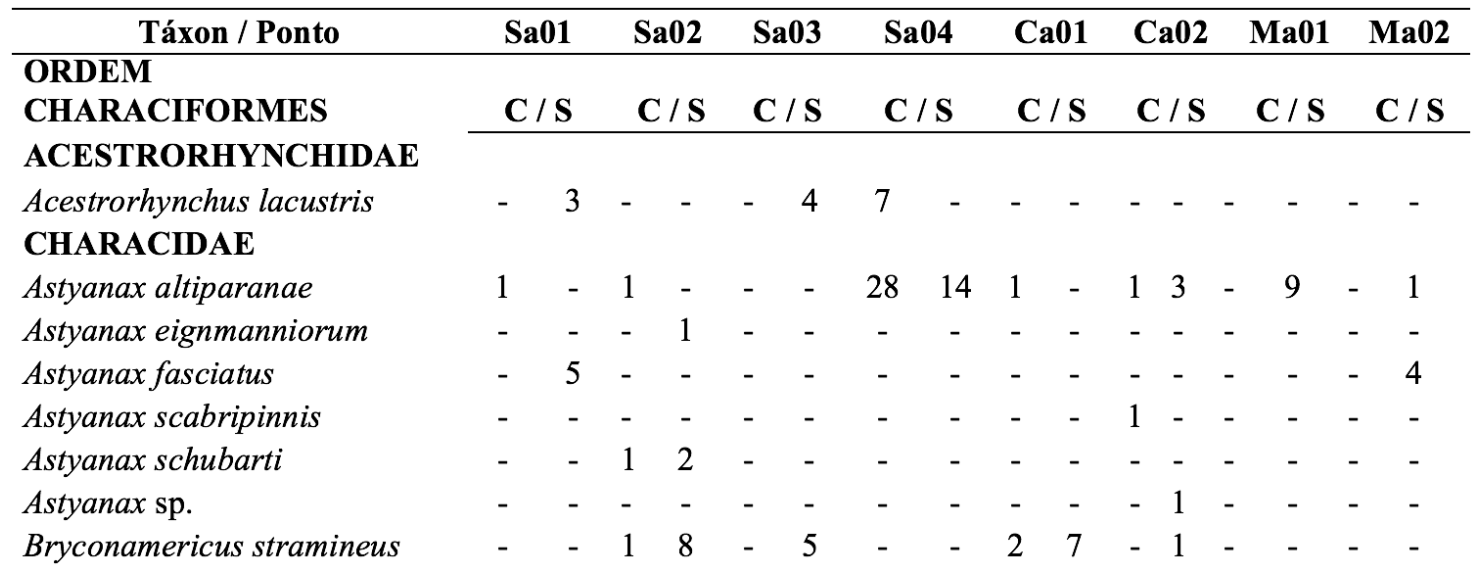




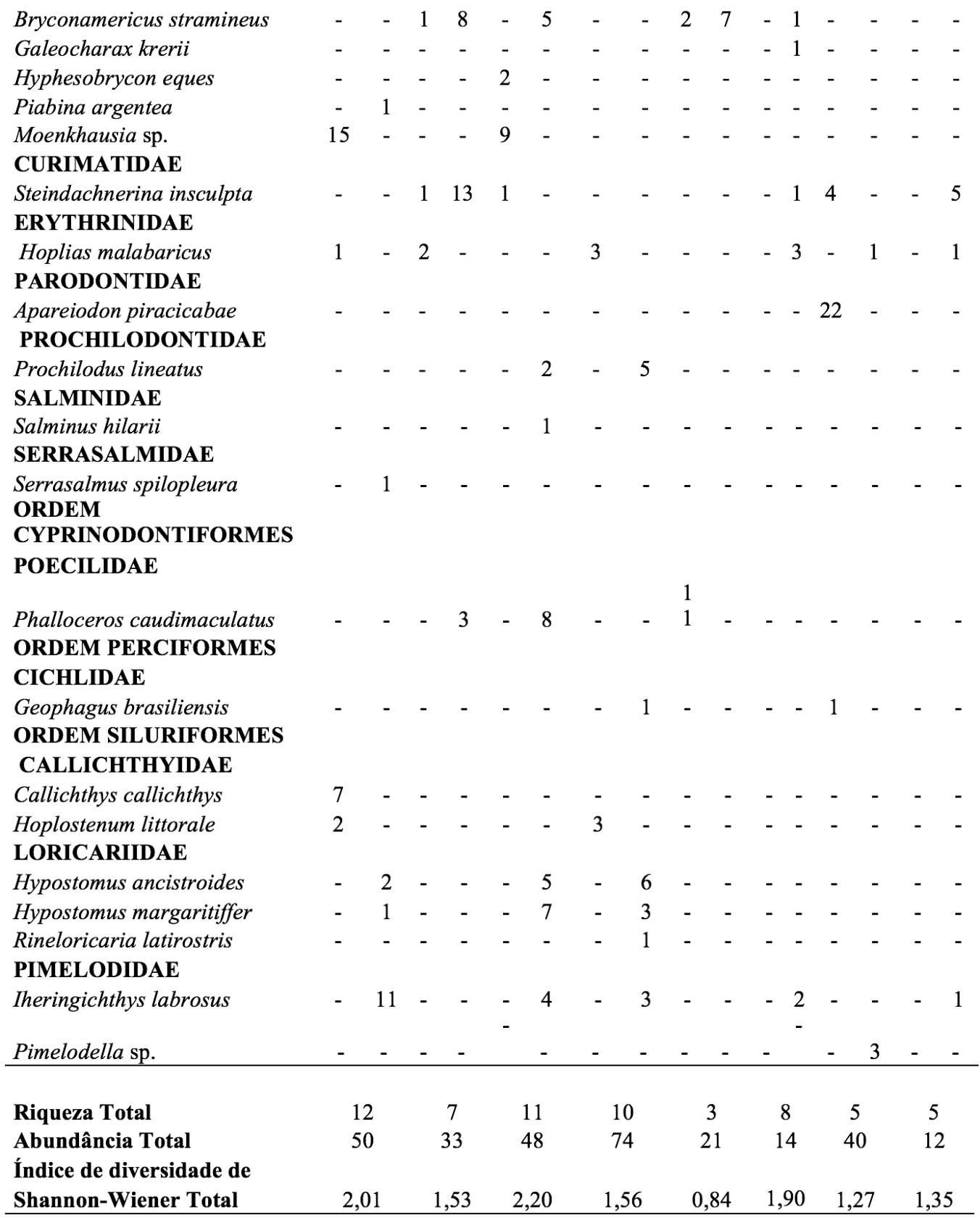
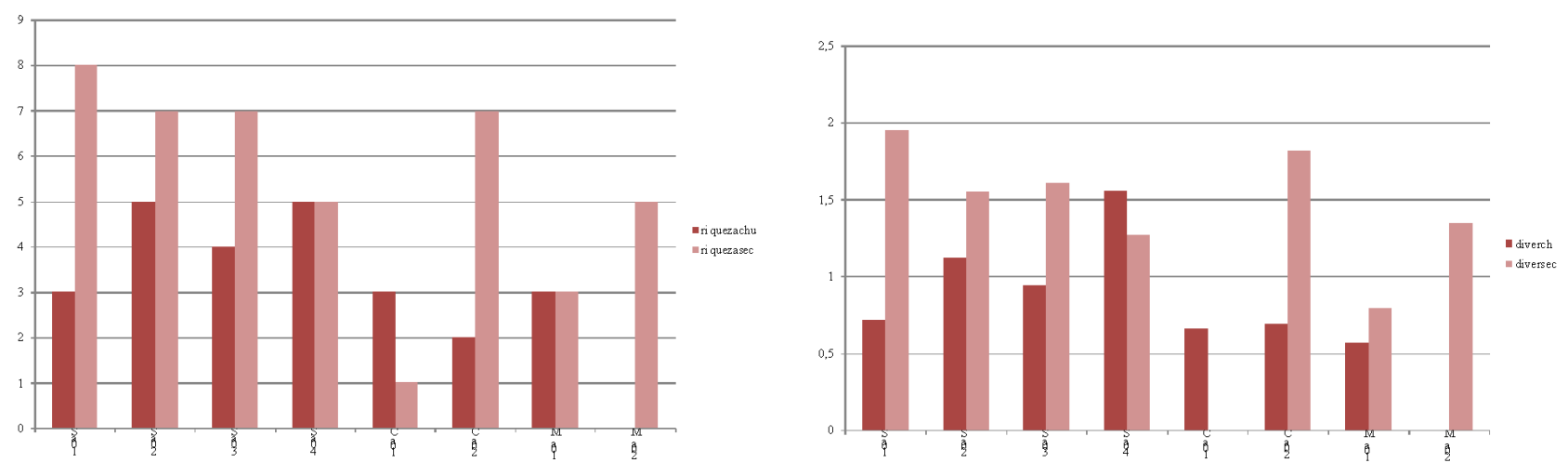

Figura 4 - Comparação dos valores de riqueza e diversidade dos pontos amostrados na bacia do rio Sarapuí e afluentes nas épocas chuvosa (chu) e seca (sec). 
De acordo com a Análise de Componentes Principais os componentes 1 e 2 (Tabela 4) explicaram $97,3 \%$ do total de variação de dados (componente $1=$ $78 \%$ e o componente $2=19,3 \%$ ). O eixo 1 foi fortemente e positivamente relacionada com um aspecto estrutural da comunidade, a abundância. No eixo 2 a variável mais importante foi a temperatura da água. Apesar da baixa correlação vale mencionar que no primeiro eixo a riqueza foi positivamente correlacionada com a abundância e no segundo a riqueza foi negativamente correlacionada com a temperatura. As variáveis que representam as condições e usos da margem não foram correlacionadas com nenhum dos dois eixos.

Tabela 4 - Valores obtidos para os dois primeiros componentes principais e seus pesos canônicos ("loadings") considerando as variáveis estudadas nos dois componentes principais e a porcentagem da variância explicada e acumulada.

\begin{tabular}{lll}
\hline VÁRIAVEIS & PC1 & PC2 \\
\hline Riqueza & $\mathbf{0 , 1 0 4}$ & $-\mathbf{0 , 1 5 2}$ \\
Abundância & $\mathbf{0 , 9 9 1}$ & 0,014 \\
Dominância & $-0,0005$ & 0,003 \\
Diversidade & 0,018 & $-0,037$ \\
Profundidade & 0,0004 & 0,061 \\
Largura & 0,064 & 0,269 \\
Pastagem & 0,005 & $-0,002$ \\
Extração de areia & 0,020 & 0,009 \\
Agricultura & $-0,025$ & $-0,0068$ \\
Temperatura da água & $-0,015$ & $\mathbf{0 , 9 4 7}$ \\
\hline \%o de Variância & $\mathbf{7 8 \%}$ & $\mathbf{1 9 , 3 \%}$ \\
\hline Variância total & & $\mathbf{9 7 , 3 \%}$ \\
\hline
\end{tabular}

De acordo com a Figura 5 e considerando o eixo 1, a maioria das amostras localizadas no rio Sarapuí (Sa01, SA02, Sa03 e SA04) distribuíram-se no lado direito do eixo, apresentando alta correlação com os valores de abundância. As amostras referentes aos afluentes Camapuã (CA01 e CA02) e Macacos (MA01 e MA02), por outro lado, localizaram-se no lado esquerdo do diagrama, apresentando-se correlação inversa com abundância. Este eixo mostra que o rio principal possui maiores abundâncias e riquezas do que os tributários.

Com relação ao eixo 2, as amostras foram divididas em dois grupos, na porção superior do digrama as amostras feitas na época chuvosa, apresentando-se positivamente correlacionadas com o eixo enquanto as amostras feitas na época seca correlacionaram inversamente com o eixo 2, estando situadas na porção inferior. Deve ser reforçado que a riqueza mostrou correlação inversa com a temperatura.

Através dessa análise foi possivel verificar que a sazonalidade influencia a ictiofauna tanto no rio principal como nos tributários e que a abundância é um descritor importante associado a riqueza e que o porte do curso dá água representado nesse estudo pela largura influência as abundâncias das espécies. Além disso, as variáveis que expressam as condições das margens e leito possui baixa influência nos descritores da comunidade utilizados.

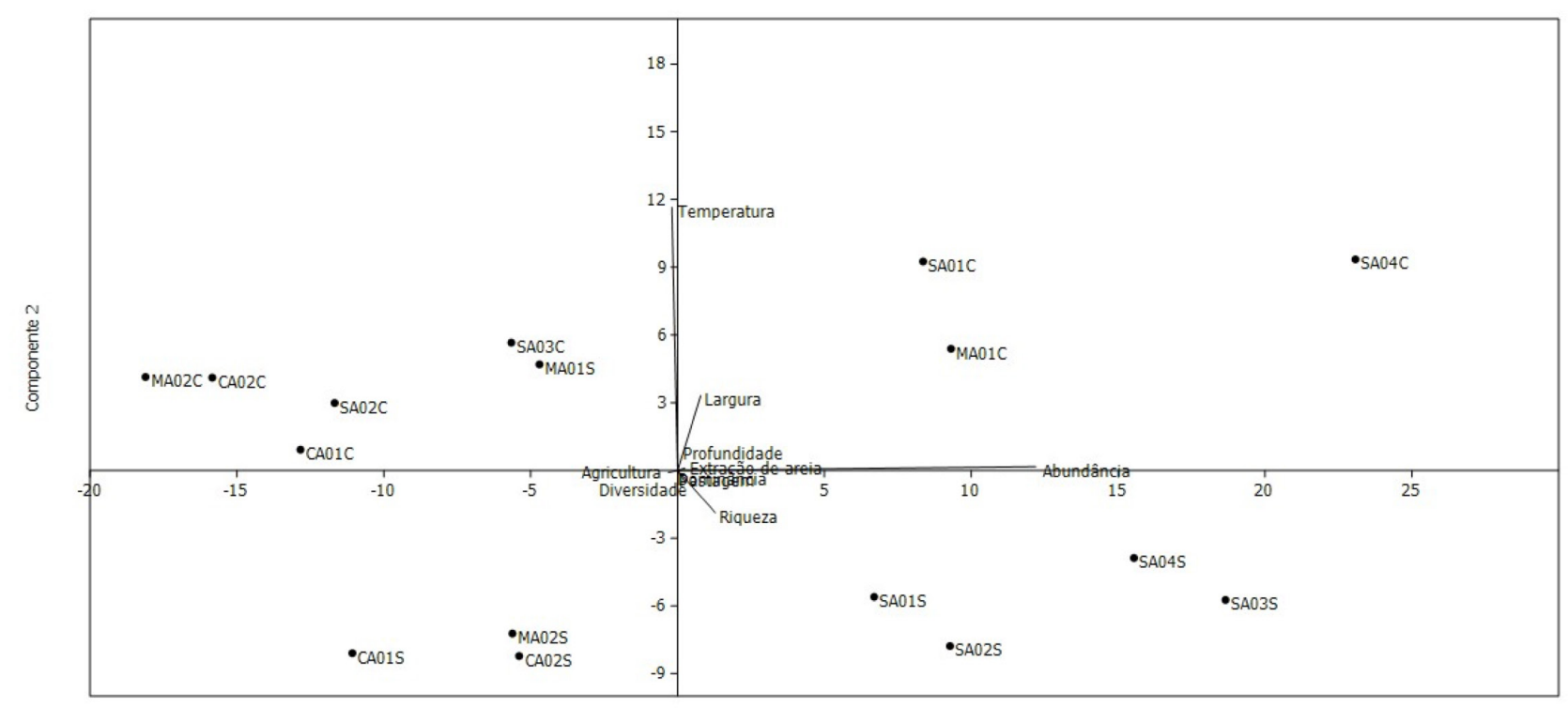

Componente 1

Figura 5 - Diagrama de dispersão da análise de componentes principais (ACP) para as variáveis abióticas e bióticas avaliadas na bacia do rio Sarapuí e afluentes. 


\section{DISCUSSÃO}

Considerando todas as bacias formadoras do rio Sorocaba, a bacia do rio Sarapuí é a mais conservada em relação ao lançamento de efluentes (Smith \& Petrere 2000). Isso é reforçado por Marciano et al. (2004) que considerou dois trechos do rio Sarapuí, através do índice de integridade biótica, como excelente e regular. Smith \& Petrere (2000) destacam as melhores condições do rio Sarapuí em relação aos outros rios da bacia, mas salientam também a extração de areia como principal impacto. Segundo dados apresentados nesse trabalho há intensa pressão decorrentes da ocupação humana nas margens, que comprometem a vegetação ripária e ocasionam o assoreamento. Teshima (2010) também constatou o mesmo cenário.

A extração de areia é um impacto relevante, já que tais práticas afetam os ambientes aquáticos por perda ou transformação de habitats (Casatti et al., 2001). De acordo com os resultados obtidos, os locais amostrados em sua grande maioria apresentaram pequena ou quase nenhuma cobertura vegetal às margens, o que causa na maioria dos trechos estudados, assoreamento. $\mathrm{O}$ assoreamento causa efeitos físicos e químicos no corpo d'água. Uma das consequências é a modificação no fluxo d'água. Essas alterações levam às mudanças na profundidade e velocidade, acarretando alterações na composição física do hábitat aquático. Como consequência na biota aquática, entre elas os peixes ocorrem mudanças estruturais na composição das espécies e a redução da diversidade. Segundo (Smith, 2003), estes impactos interferem na riqueza e diversidade da ictiofauna. A inexistência de mata ciliar reduz a diversidade de peixes, em virtude da redução da entrada de suprimento orgânico, de hábitats para alimentação, desova e abrigo (Barrella et al., 2000).

Outras perturbações são relativas a mudanças na estrutura trófica da comunidade de peixes, diminuindo a abundância relativa de insetívoros, bentônicos e herbívoros. O aumento da sedimentação reduz a quantidade de alimentos disponíveis para os peixes, modificando os locais utilizados para as desovas, acarretando em mudanças comportamentais dos peixes, no aumento da mortalidade de ovos e larvas, afetando inclusive o desenvolvimento e a sobrevivência delas (Berkman \& Rabeni, 1987).

A maioria dos pontos possui ausência ou vegetação ripária degradada, e como consequência o assoreamento do leito é facilmente percebido, provavelmente acarretando na perda dos abrigos, o que pode estar afetando as comunidades de peixes. Segundo Pastore \& Mioto (2000) alterações nos corpos d'água devido ao carreamento de sedimento, compromete a qualidade da água sendo o efeito ecológico drástico. Segundo Gorman et al. (1978) poucas espécies estão adaptadas às novas condições.

O presente estudo procurou contribuir com informações acerca das assembleias de peixes da bacia do rio Sarapuí. Considerando os trabalhos realizados por Smith (1999), Smith et al. (2007) e Smith et al. (2009) em dois pontos do rio Sarapuí e um ponto no ribeirão dos Macacos, este trabalhou adicionou 13 espécies ao que se conhecia até o momento na bacia do rio Sarapuí. Com relação às espécies exóticas, apesar dos trabalhos não terem documentado nenhuma, elas estão presentes na bacia do rio Sarapuí, tendo sido apontado por pescadores a presença da tilápia, provavelmente Oreochromis niloticus e Tilapia rendalli.

Os resultados deste estudo sugerem uma assembleia com alta riqueza de espécies e com um padrão documentado por outros trabalhos, desenvolvidos no Alto Paraná tal como Langeani et al. (2007) que afirma que os Siluriformes e Characiformes respondem por cerca de $80 \%$ das espécies e compõem os grupos dominantes. O mesmo foi verificado para o estado de São Paulo (Oyakawa \& Menezes, 2011) e para a bacia do rio Sorocaba (Smith et al., 2007, 2009). Segundo AraújoLima et al. (1995), em ambientes distintos podem ser identificadas diferentes proporções entre as espécies de Characiformes e Siluriformes. Verifica-se que nas margens há o predomínio de Characiformes enquanto que no canal do rio predominam os Siluriformes.

Entre as famílias, a Characidae foi a mais importante em relação ao número de espécies capturadas (11) seguida de Loricariidade (3). Esse resultado corrobora com o padrão apresentado por Oyakawa \& Menezes (2011) que evidenciaram 83 espécies de Characidae e 81 de Loricariidae para o estado de São Paulo. Resultados similares em relação a abundância e distribuição foram obtidos por Smith (1999), Smith et al. (2007), Smith et al. (2009), Teshima (2010) e Cetra et al. (2012).

Entre as espécies citadas na presente lista do Estado de São Paulo, nenhuma das inventariadas nesse estudo é citada. A não existência de espécies que se encontram nas listas oficiais se deve ao presente estudo não ter sido focado em riachos, ambientes onde são encontrados a maioria das espécies de peixes ameaçadas do estado de São Paulo (Oyakawa et al., 2009). É reconhecido que é nas regiões de cabeceira, onde habitam diversas espécies de pequeno porte, de distribuição geográfica restrita e endemismo elevado, com ocorrência exclusiva em uma localidade ou região particular (Menezes, 1998; Castro, 1999) que se concentram a maioria das ameaçadas. Complementando, grandes peixes 
migradores como o jaú (Zungar jahu) e o pintado (Pseudoplatystoma corruscans) já documentados em décadas passadas para a bacia do rio Sorocaba e atualmente não mais encontrado podem integrar espécies extintas localmente ou raras.

Deve ser salientada a primeira ocorrência para a bacia do rio Sorocaba e também para a bacia do rio Sarapuí, da espécie Rhinodoras dorbignyi. Vasconcelos et al. (2014) considera que esta espécie pode executar migração reprodutiva de curta distância. No Brasil, esta espécie ocorre na bacia Paraná-Paraguai (Graça \& Pavanelli, 2007), que é severamente impactado por barramentos. Rhinodoras dorbignyi foi caracterizada como uma espécie rara em comparação a outras espécies dessa bacia (Fagundes et al., 2007). Esta espécie é dificil de capturar e o que se conhece sobre a sua biologia e ecologia é incipiente. Tais considerações podem explicar o porquê dessa espécie não ter sido capturada anteriormente pela pesca experimental nos diferentes trabalhos realizados na bacia.

A alta riqueza encontrada na bacia do rio Sarapuí deve-se a ampla amostragem da bacia, contemplando coletas nas cabeceiras, no rio principal e seus tributários e também em ambientes importantes como as lagoas marginais. Estas inclusive devem ser conservadas e destacadas a sua importância como local que detém relativa riqueza de espécies e por servir de locais de abrigo e desenvolvimento para alevinos de espécies como o curimbatá. Smith \& Barrella (2000) salientaram a importância das lagoas marginais para o rio Sorocaba, o que também foi verificado pelo presente estudo, para o rio Sarapuí. Os maiores valores de riqueza, abundância e diversidade encontrados nas localidades avaliadas, seja pela adição ou substituição de espécies, podem ser explicados pela maior complexidade e quantidade de hábitats (Angermeier \& Schlosser 1989, Schlosser, 1990) que esses locais possuem. É possível verificar que o maior esforço foi realizado no rio Sarapuí em seu trecho médio, incluindo seus principais tributários. O Alto Sarapuí e seu trecho baixo, além de outros tributários, requerem maior esforço de coleta, pois são áreas pouco estudadas e que podem apresentar novas espécies para a bacia.

As espécies Astyanax altiparanae, Astyanax fasciatus, Steindachnerina insculpta, Hoplias malabaricus, Hypostomus ancistroides, Iheringichthys labrosus, Geophagus brasiliensis e o gênero Phalloceros foram amplamente capturadas, refletindo suas amplas distribuições geográficas como salientado por Buckup et al. (2007) e Oyakawa \& Menezes (2011) para o Brasil e estado de São Paulo. Ao todo 5 espécies não possuem seu estado taxonômico identificado. Estes dados refletem a situação que mesmo a Bacia do Alto Paraná sendo bem estudada, comparada com outras bacias da América do Sul, muitas espécies ainda podem ser descritas com os avanços dos trabalhos principalmente nas cabeceiras e tributários de rios principais (Langeani et al., 2007).

A ocorrência de espécies em determinado ambiente aquático está relacionada às interações interespecíficas e mantém constante relação entre si (Súarez \& Petrere 2003; Santos, 2009). A ausência ou a presença de espécies em um determinado ambiente dependem ainda da alimentação, refúgio e reprodução que esse próprio ambiente fornece (Bennemann et al., 2000). Sendo assim, algumas considerações a respeito de determinadas espécies se tornam relevante. No ribeirão do Camapuã (CA01 e CA02), a espécie Bryconamericus stramineus foi abundante e capturada próximo da vegetação, enquanto que no ribeirão dos Macacos (MA01 e MA02) a espécie Apareiodon piracicabae foi predominantemente encontrada em áreas marginais de fundo arenoso. Isso reforça a importância dos tributários e também das áreas marginais onde muitas espécies de pequeno porte como essas exploram os recursos existentes.

No rio Sarapuí, a espécie Astyanax altiparanae está amplamente distribuída, sendo a mais representativa na composição das espécies de peixes. Esta espécie é caracterizada por indivíduos com alta mobilidade, ativos, sendo considerados forrageiros. São flexíveis quanto aos hábitos alimentares, habitam as margens do rio e suas lagoas marginais. Estas espécies nadam entre a meia água e a superfície (Smith \& Petrere, 2007). Além dessas espécies, é comum a espécie Steindachnerina insculpta, espécie de hábitos bentônicos, ocorrendo em ambientes lênticos (Smith, 2003).

Considerada como indicadora de qualidade ambiental (Cetra, 2003), a espécie Salminus hilarii foi capturada no rio Sarapuí no ponto SA03. Esta espécie é migradora e se reproduz nas estações chuvosas, com período reprodutivo curto, ausência de cuidado parental, corpo de porte médio a grande, alta fecundidade e ovos livres (Sato et al., 2003). Também pode ser considerada importante do ponto de vista de integridade ambiental a espécie Prochilodus lineatus considerada migradora e capturada no médio e baixo rio Sarapuí, nos pontos SA03 e SA04.

A variação da riqueza demonstrou que as comunidades são afetadas pelas mudanças sazonais que implicam na alteração dos parâmetros abióticos, que interferem diretamente na dinâmica populacional das espécies. Neste estudo, setembro/2010 apresentou as médias mais baixas de temperatura, bem como a menor riqueza e abundância de espécies. Santos \& Caramaschi (2011) também verificaram diferenças na temperatura entre a época seca e chuvosa, sendo esta variável uma das que mais correlacionaram 
com abundância dos peixes. Em rios tropicais a comunidade de peixes é afetada pelas mudanças sazonais, já que esta expande o ecosistema aquático da época seca para a chuvosa (Santos \& Caramaschi, 2011). Variações na abundância em decorrência da sazonalidade podem refletir mudanças na estrutura da assembleia de peixes.

Ferreira \& Santos (2006) afirmam que a maior ocorrência de indivíduos jovens e a maior proporção de indivíduos reprodutivos ocorrem entre novembro e março, relacionando a reprodução e o recrutamento ao período chuvoso. Sendo assim quando há reprodução e recrutamento alto na época chuvosa, a época seca seguinte à abundância é maior e vice-versa. Segundo Abdon (2004) essa situação interfere na diversidade de peixes, causando também outras alterações ecológicas que a modificam naturalmente ao longo do curso do rio. Além disso, dificulta a utilização dos petrechos de captura resultando em uma riqueza de espécies menor do que na época seca. Isso foi verificado pelo presente estudo, onde dos 8 pontos estudados 5 apresentaram maior riqueza na época seca.

Apesar da presença de mata ciliar, os pontos de coletas amostrados apresentaram próximo à margem áreas de pastagem, agricultura e vestígios de antigas extrações de areia. Nos 4 pontos do rio Sarapuí, notouse a presença de galhos e troncos de árvores caídos em seu leito, favorecendo o abrigo para as espécies de peixes. Os pontos CA01 e CA02 não apresentaram alterações antrópicas consideráveis, enquanto que os pontos MA01 e MA02 são caracterizados por ausência de mata ciliar.

Atravéz da análise de componentes principais foi possivel verificar que a sazonalidade influencia a ictiofauna tanto no rio principal como nos tributários e que a abundância é um descritor importante associado a riqueza e que o porte do curso dá água representado nesse estudo pela largura influência as abundâncias das espécies. Além disso, as variáveis que expressam as condições das margens e do leito apresentam baixa influência nos descritores da comunidade utilizados. Além disso, o efeito da sazonalidade sobre a abundância foi mais evidente no rio Sarapuí de maior porte do que nos seus tributário. Deve ser ressaltado que as variáveis profundidade, largura e atividades presentes na área de preservação permanente (pastagem, extração de areia e agricultura) não apresentaram correlação significativa com os eixos da ACP. Sendo assim, pode-se supor que outros fatores tais como a biogeografia e fatores historicos (Ribeiro, 2006), fatores físicos e químicos (Casatti et al., 2006) e interações bióticas (Taylor \& Warren, 2001) os mais relevantes para a estruturação da assembléia de peixe. Takahashi et al. (2013) afirmam que pequena variação sazonal na assembleia de peixes tem sido verificadas nos trechos altos dos rios. Segundo esses autores a conectividade e a migração sazonal influênciam as diferenças na assembleia em diferentes épocas do ano. As diferenças sazonais verificadas na estrutura da assembleia de peixes observadas nos trechos maiores parece ser fortemente influênciada pela migração dos peixes, oriundos de rios maiores a jusante.

\section{AGRADECIMENTOS}

Agradecemos à Geralda Dias Rocha, Francisco Freire Rocha e Márcio Aparecido pelo auxilio nas coletas.

\section{REFERÊNCIAS BIBLIOGRÁFICAS}

Abdon, M.M. 2004. Os impactos ambientais no meio físico - erosão e assoreamento na bacia hidrográfica do rio Taquari, MS, em decorrência da pecuária. Tese de doutorado. Centro de Recursos Hídricos e Ecologia Aplicada da Escola de Engenharia de São Carlos -Universidade de São Paulo, São Carlos: EESC/USP. 274p.

Agostinho, A.A, Thomaz, S.M. \& Gomes, L.C. 2005. Conservação da biodiversidade em água continentais do Brasil. Megadiversidade. 1(1): 70-78.

Angermeier, P.L. \& Schlosser, I.J. 1989. Species area relationship for stream fish. Ecology. 70(5): 1450-1462.

Araújo, F.G., Pinto, B.C.T. \& Teixeira, T.P. 2009. Longitudinal patterns of fish assemblages in a large tropical river in southeastern Brazil: evaluating environmental influences and some concepts in river ecology. 2009. Hydrobiologia 618:89-107 DOI 10.1007/s10750-008-9551-5.

Barrella, W. 1989. Estrutura da comunidade de peixes da bacia do rio Jacaré Pepira (SP) em diferentes biótopos. Tese de Mestrado. UNICAMP. 198p.

Barrella, W.; Petrere, Jr. M.; Smith,W.S. \& Montag, L.F.A. 2000. As relações entre as matas ciliares, os rios e os peixes. In Rodrigues RR, Leitão Filho HF. Matas Ciliares: conservação e recuperação. p. $187-207$.

Bennemann, S.T., Shibatta, O.A., Garavello, J.C. 2000. Peixes do rio Tibagi: uma abordagem ecológica. Londrina, UEL. 64p.

Buckup, P.A.; Menezes, N.A. \& Ghazzi, M.S. (eds.). 2007. Catálogo das espécies de peixes de água doce do Brasil. Série livros 23. Museu Nacional. Universidade Federal do Rio de Janeiro, Rio de Janeiro. 195p. 
Berkman, H.E. \& Rabeni, C.F. 1987. Effect os siltation on stream fish communities. Environmental Biology of Fishes. 18(4): 285-294.

Buss, D.F., Oliveira, R.B. \& Baptista, D.F. 2008. Monitoramento biológico de ecossistemas aquáticos continentais. Oecologia Brasiliensis. 12(3): 339-345.

Cassati, L., Langeani, F. \& Castro R.M.C. 2001. Peixes de riacho do parque estadual Morro do Diabo, bacia do Alto rio Paraná, SP. Biota Neotropica. 1(1): 1-15.

Cassati, L., Langeani, F., Silva, A.M. \& Latini, J.D. 2006. Stream fish, water and habitat quality in a pasture dominated basin, southastern Brazil. Brazilian Journal of Biology. 66: 681-696.

Castro, R.M.C. 1999. Evolução da ictiofauna de riachos sul-americanos: padrões gerais e possíveis processos causais. Pp. 139-155 In: E.P. Caramaschi, R. Mazzoni, C.R.S.F. Bizerril \& P.R. Peres- Neto (Eds.). Ecologia de peixes de riachos. Série Oecologia Brasiliensis, vol. 7, PPGE-UFRJ, Rio de Janeiro, 260 pp.

Cetra, M. 2003. Caracterização da assembléia de peixes da bacia do Rio Corumbataí (SP). Tese de Doutorado. Escola de Engenharia de São Carlos, Universidade de São Paulo, São Carlos. 423p.

Cetra, M., Barrella, W., Langeani Neto, F., Martins, A. G., Mello, B. J. \& Almeida, R. S. 2012. Fish fauna of headwater streams that cross the Atlantic Forest of south São Paulo state. Check List. 8(3): 421-425

Castro, R.M.C. \& Menezes, N.A. 1998. Estudo diagnóstico da diversidade de peixes do Estado de São Paulo. In Biodiversidade do Estado de São Paulo, Brasil: síntese do conhecimento ao final do século XX, 6, vertebrados (R.M.C. Castro, ed.). WinnerGraph \& FAPESP, São Paulo, p.1-13.

Dala-Corte, R.B., Franz, I., Barros, M.P. \& Ott, P.H. 2009. Levantamento da ictiofauna da Floresta Nacional de Canela, na região superior da bacia hidrográfica do rio Caí, Rio Grande do Sul, Brasil. Biota Neotropica. 9(2): 221-226.

Digby, P.G.N. \& Kempton, R.A. 1987. Multivariate analysis of ecological communities. London: Chapman \& Hall, 206p.

Fagundes, C. K.; Behr, E. R.; Kotzian, C. B. 2007. Alimentação de Rhinodoras dorbignyi (Kröyer, 1855) (Siluriformes: Doradidae) no rio Ibicuí, Rio Grande do Sul, Brasil. Acta Scientiarium Biological Sciences, Maringá, v. 27, n. 4, p. 365-369.

Felipe, T.R.A. \& Súarez, Y.R. 2010. Characterization and influence of environmental factors on stream fish assemblages in two small urban sub-basins, Upper Paraná River. Biota Neotropica. 10(2): 143-151.

Ferreira, C.P. \& CasatTi, L. 2006. Influência da estrutura do hábitat sobre a ictiofauna de um riacho em uma micro-bacia de pastagem, São Paulo, Brasil. Revista Brasileira de Zoologia. 23 (3): 642-651.

Ferreira, M.P. \& Santos, A.C.A. 2006. Variação sazonal, recrutamento e hábitos alimentares de Tetragonopterus chalceus Spix \& Agassiz, 1829 (Characiformes, Characidae), no Rio Santo Antônio, Bahia, Brasil. Revista Brasileira de Zoociências. 8 (2): 117-123.

Gorman, O.T. \&. KARR, J.R. 1978. Habitat structure and stream fish communities. Ecology. 59(3): 507-15.

Graça, W. J.; Pavanelli, C. S. 2007. Peixes da planície de inundação do Alto Rio Paraná e áreas adjacentes. Maringá: EDUEM, 168 p.

Hammer, O., Harper, D.A.T. \& Ryan, P.D. 2001. PAST: Paleontological statistics software package for education and data analysis. Palaeontologia Electronica. 4(1): 1-9.

Hoelzl, U. 2007. River habitat monitoring and assessment in Germany. Environmental Monitoring and Assessment, 127p.

Hoffman, A.C., Orsi, M.L. \& Shibatta, O.A. 2005. Diversidade de peixes do reservatório da UHE Escola Engenharia Mackenzie (Capivara), Rio Paranapanema, bacia do alto rio Paraná, Brasil, e a importância dos grandes tributários na sua manutenção. Iheringia, Ser. Zool. Poto Alegre. 95(3): 319-325.

INSTITUTO BRASILEIRO DE GEOGRAFIA E ESTATÍSTICA. Cidades. 2009 [acesso em 02 out 2010]. Disponível em: http://www.ibge.gov.br/ cidadesat/topwindow.htm?1

INSTITUTO BRASILEIRO DE GEOGRAFIA E ESTATÍSTICA. Biblioteca. [acesso em 02 out 2010] Disponível em: http://biblioteca.ibge.gov. br/visualizacao/mapas/GEBIS\%20-\%20RJ/SF23-Y-C.jpg

Langeani, F., Castro, R.M.C., Oyakawa, O.T., Shibatta, O.A., Pavanelli, C.S. \& Casatti, L. 2007. Diversidade da ictiofauna do alto Rio Parana: composição atual e perspectivas futuras. Biota Neotropica. 7(3): 1-13.

Manly, B.J. 1986. Multivariate statistical methods: a primer. London: Chapman \& Hall, 281p.

Menezas, N.A. 1998. Padrões de distribuição da biodiversidade da mata atlântica do sul e sudeste brasileiro: peixes de água doce. In: Base de Dados Tropical (ed.). Biodiversity Patterns of South and Southeast Atlantic Rain Forest. http://www.bdt.org. $\mathrm{br} / \mathrm{bdt} /$ workmatasud/peixes.

Marciano, F.T., F.H. Chaudhry \& M.C.D. de B. Ribeiro. 2004. Evaluation of the index of biotic integrity in the Sorocaba River Basin (Brazil, SP) based on 
fish communities. Acta Limnologica Brasileira. 16(3): 225-237.

Marinho, R.S.A., Souza, J.E.R.T., Silva, A.S. \& Ribeiro, L.L. 2006. Biodiversidade de peixes do semiárido paraibano. Revista de Biologia e Ciência da Terra.1: 112-121.

Odum, E.P. \& Barrett, G.W. 2011. Fundamentos de Ecologia. São Paulo: Cengage Learning. p.424-430.

Oleriano, E.S. \& Dias, H.C.T. 2007. A dinâmica da água em microbacias hidrográficas reflorestadas com eucalipto. Taubaté, Brasil, IPABHi. p. 215-222.

Oyakawa, O.T.; Menezes, N.A.; Shibatta. O.A.; Lima, F.C.T.; Langeani, F.; Pavanelli, C.S.; Nielsen, D.T.B.; Hilsdorf, A.W.S. 2009. Peixes de água doce. In: Bressan, P.M.; Kierulff, M.C. \& Sugieda, A.M. (Orgs), Fauna ameaçada de extinção no Estado de São Paulo: Vertebrados. São Paulo, Fundação Parque Zoológico de São Paulo e Secretaria do Meio Ambiente.

Oyakawa, O.T. \& Menezes, N.A. 2011. Checklist dos peixes de água doce do Estado de São Paulo, Brasil. Biota Neotropica. 11:(1a): 1-13.

Pastore, E.L. \& Mioto, J.A. 2000. Impactos ambientais em Mineração com ênfase à drenagem mineira ácida e transporte de contaminantes. Revista Latino-Americana de Geotecnia. 23(1): 33-53.

Pozzebon, E.J. 2000. Simulação de escoamento em Microbacia Hidrográfica utilizando sistemas de informações geográficas e de modelagem hidrológica. Tese de Doutorado. Universidade de São Paulo - USP, Piracicaba - SP. 234p.

Ribeiro, A.C. 2006. Tectonic history and the biogeography of the freshwater fishes from the coastal drainages of eastern Brazil: an example of faunal evolution associated with a divergent continental margin. Neotropical Ichthyology. 4: 225-246.

Santos, V.K. 2009. Uma generalização da distribuição do índice de diversidade generalizada por Good com aplicação em Ciências Agrárias. Tese de Dissertação de Mestrado. Universidade Federal Rural de Pernambuco. 57p.

Santos, D.A. Dos; Molineri, C; Reynaga, M.C. \& Basualdo, C. 2011. Which index is the best to assess stream health? Ecological Indicators. 11: 582-589.

Santos, A.C.A. \& Caramaschi, E.P. 2011. Temporal variation in fish composition and abundance in a perennial tributary of the rio Paraguaçu, a littleknow drainage in the Brazilian semi-arid region. Neotropical Ichthyology. 9(1): 153-160.

Sato, Y.; Fenerich-Verani, N.; Nuner, A.P.O.; Godinho, H.P.; Verani, J.R. 2003. Padrões reprodutivos de peixes da bacia do São Francisco. In: GODINHO,
H.P. e GODINHO, A.L. Águas, peixes e pescadores do São Francisco das Minas Gerais. Belo Horizonte: PUCMinas. 458p.

Schlosser, I.J. 1990. Environmental variation, life history attributes, and community structure in stream fish: implications for environmental management and assessment. Environ. Manage. 14: 621-628.

Smith, W. S. 1999. A estrutura da comunidade de peixes da bacia do rio Sorocaba em diferentes situações ambientais. Tese de Mestrado. Universidade de São Paulo, São Carlos. 121p.

Smith, W.S. \& Barrella, W. 2000. The ichthyofauna of the marginal lagoons of the sorocaba river, SP, Brazil: Composition, abundance and effect of the anthrogenic actions. Brazilian Journal of Biology. 60(4): 627-632.

Smith, W.S. \& Petrere, JR. M. 2000. Caracterização Limnológica da bacia de drenagem do rio Sorocaba, São Paulo, Brasil. Acta Limnologica Brasiliensia. (12): 15-27.

Smith, W.S. 2003. Os Peixes do Rio Sorocaba: A história de uma bacia hidrográfica, $1^{\text {a } e d . ~ S o r o c a b a: ~}$ TCM. 160p.

Smith, W.S. \& Petrere, JR.M. 2007. Fish, Itupararanga Reservoir, Sorocaba River Drainage, São Paulo, Brazil. Check List. 3(2): 282-286.

Smith, W.S., Petrere Jr, M. \& Barrella, W. 2007. Fish, Sorocaba river sub-basin, state of São Paulo, Brazil. Check List. 3(3): 282-286.

Smith, W.S., Petrere Jr, M. \& Barrella, W. 2009. The fish community of the Sorocaba River Basin in different habitats (State of São Paulo, Brazil). Brazilian Journal of Biology. 69(4): 1015-1025.

Súarez, R.Y. \& Petrere Jr. M. 2003. Associações de espécies de peixes em ambientes lóticos da bacia do rio Iguatemi, Estado do Mato Grosso do Sul. Acta Scientiarum. Biological Sciences. 25(2): 361-367.

Takahashi, E.L.H., Rosa, F.R.T., Langeani, F. \& Nakaghi, L.S.O. 2013. Spatial and seasonal patterns in fish assemblage in Córrego Rico, upper Paraná River Basin. Neotropical Ichthyology. 11(1): 133-152.

Taylor, C.M. \& Warren, M. L. 2001. Dynamics in species composition of stream fish assemblages: Environmental variability and nested subsets. Ecology. (82): 2320-2330.

Teshima, F.A. 2010. Caracterização da ictiofauna e avaliação dos atributos ambientais em trechos superiores do rio Sarapuí (Piedade, SP). [Monografia]. Universidade Federal de São Carlos. 69p.

Uieda, V.S. \& Castro, R.M.C. 1999. Coleta e fixação de peixes de riachos. In: Caramaschi, E. P.; Mazzoni, 
Nascimento, M. B. \& Smith, W. S. (2016). A ictiofauna do rio Sarapuí, São Paulo, Brasil.

R.; Peres-Neto, P. R. (Eds.). Ecologia de Peixes de Riachos, Série Oecologia Brasiliensis. Vol. VI. PPGE-UFRJ, Rio de Janeiro, Brasil. 1-22pp.

Vannote, R.L., Minshall, G.W., Cummins, K.W., Sedell, J.R. \& Cushing, C. E. 1980. The river continuum concept. Can. J. Fish. Aquat. Sci. (37): 130-137.

Vasconcelos, L. P.; Alves, D. C.; Gomes, L. C. 2014. Fish reproductive guilds downstream of dams. Journal of Fish Biology, Malden, v. 85, p. 1489-1506.

Walsh, C.J., Allison, H.R., Feminella, J.W., Cottingham, P.D., Groffman, P.M., II, R.P.M. 2005. The urban stream syndrome: current Knowledge and the Search for a Cure. Journal of the North American Benthological Society. (24): 706-723.

Submetido: Junho/2014

Revisado: Julho/2016

Aceito: Julho/2016 